\title{
Association of immune-related adverse events with immune checkpoint inhibitor efficacy: real or imaginary?
}

\author{
Koji Haratani, Hidetoshi Hayashi ${ }^{*}$ and Kazuhiko Nakagawa
}

Keywords: Immune-related adverse event, Immune checkpoint inhibitor, Immunotherapy, PD-1, PD-L1, Metaanalysis, Systematic review

\section{Background}

Immune checkpoint inhibitors (ICIs) have become a standard of care for various types of cancer as a result of their demonstrated long-term survival benefit [1]. ICI therapy reinvigorates immune cells to achieve such treatment efficacy, but this mechanism of action can also give rise to autoimmune-like side effects known as immune-related adverse events (irAEs) [2]. The spectrum of irAEs is broad in that they can involve various organs, and, although they are usually mild or manageable, they can be lifethreatening [2]. However, of note, evidence has suggested that these irAEs may be an early sign of ICI treatment success [3]. Indeed, numerous studies have revealed a positive association of irAEs with tumor response or extended survival in patients receiving ICI treatment. The fact that not all studies have confirmed such an association, however, has left the nature of the relation between irAEs and ICI treatment efficacy unclear [3].

In an article published recently in this journal, Zhou et al. approached this important clinical question by undertaking a comprehensive systematic review and well-designed meta-analysis [4].

This comment refers to the article available at https://doi.org/10.1186/ s12916-020-01549-2.

* Correspondence: hidet31@med.kindai.ac.jp

Department of Medical Oncology, Kindai University Faculty of Medicine,

377-2 Ohno-higashi, Osakasayama, Osaka 589-8511, Japan

\section{Systematic review identifies $\mathbf{3 0}$ articles for meta- analysis of PFS and OS}

For their meta-analysis, Zhou et al. considered only studies that reported hazard ratios for progression-free survival (PFS) or overall survival (OS) in patients with irAEs compared with those without irAEs, resulting in the inclusion of 30 original articles for the analysis. These 30 articles consisted mostly of studies that evaluated patients with non-small cell lung cancer or melanoma who were treated with inhibitors of programmed cell death-1 (PD-1) or its ligand PD-L1, although patients with other cancer types or those treated with ipilimumab, an antibody to cytotoxic $\mathrm{T}$ lymphocyte antigen-4 (CTLA-4), were also included. The metaanalysis evaluated potential publication bias by funnel plot-based analyses with Egger's test and the trim-andfill method. It examined between-study heterogeneity by applying the $\chi^{2}$ test and $I^{2}$ statistic, with a randomeffects model being adopted when significant heterogeneity was observed, and sensitivity analyses were also performed.

\section{Positive association of irAEs with ICI treatment efficacy}

Vigorous investigation of potential publication bias did not reveal serious bias in this study. The meta-analysis showed that both PFS and OS were significantly longer in patients with irAEs than in those without them. The hazard ratios were 0.52 and 0.54 , respectively, meaning that the association was also clinically significant, 
although significant heterogeneity was detected by the $\chi^{2}$ test and $I^{2}$ statistic ( $p<0.01$ and $50-75 \%$, respectively). The authors evaluated the quality of each included original study with the Newcastle-Ottawa Scale [5], finding that quality was not clearly problematic (average and median scores of 5.47 and 5, respectively), even though most included studies were retrospective. One of the most important methodological issues in this type of study is how to alleviate lead-time bias, given that the exposure (irAEs) can fluctuate based on outcome (the better the treatment efficacy, the higher the frequency of irAEs). A landmark analysis is thus important to minimize this lead time bias [3, 6]. Of note, predefined sensitivity analysis including only studies that adopted landmark analysis also confirmed the positive association between irAE occurrence and better treatment outcome. In addition, predefined sensitivity analysis including only studies that performed multivariable analysis or those with a large sample size $(N \geq 100)$ did not alter the main conclusions of this meta-analysis.

A subgroup analysis according to irAE type or severity suggested that only cutaneous or endocrine irAEs as well as mild irAEs (grade 1 or 2) can be associated with better treatment outcomes. This was possibly due to the unnecessity for permanent discontinuation of ICI treatment [7] or for high-dose systemic glucocorticoid treatment, which can counteract antitumor immunity induced by ICI therapy [8]. These findings require a further clinical examination for verification, however. The subgroup analysis also did not reveal a difference among cancer types in the association of irAEs with treatment outcomes. With regard to ipilimumab treatment, such an association was not apparent, possibly as a result of the small number of studies included.

\section{This meta-analysis provides a limited generalizability}

There is a possible concern with the application of the findings of this meta-analysis to clinical practice. Most of the included studies were retrospective observational studies, which may limit the quality of the analysis. Although meta-analyses in general provide a high level of evidence, they are dependent on the quality of the included studies. In particular, meta-analyses based on observational studies should be interpreted with caution. In this regard, the GRADE approach has been proposed as a means of evaluating the quality of meta-analyses. The quality of the present study can be estimated as low to moderate, given the possible risk of bias or inconsistency $[9,10]$, and its conclusions thus might not be perfectly generalizable for our clinical practice. However, given this type of investigation cannot be designed as an intervention study because irAEs are not experimental, a meta-analysis of observational studies may provide the best evidence possible.

\section{Future directions}

Combination treatment with an ICI and other modalities including cytotoxic chemotherapy, molecularly targeted therapy, or localized therapy such as surgery or radiation is emerging as a new standard of care for many types of malignancies [1]. Whether the occurrence of irAEs during such combination therapy is associated with or predictive of long-term survival outcome is therefore of great clinical interest. Well-designed prospective observational studies that adopt both landmark analysis and multivariable analysis are needed to address this issue.

\section{Conclusions}

The comprehensive systematic review and well-designed meta-analysis performed by Zhou et al. have provided important evidence for a positive association of irAE occurrence with a better outcome of ICI treatment-in particular, for the treatment of solid cancers with PD-1 or PD-L1 inhibitors.

\section{Acknowledgements}

Not applicable.

\section{Authors' contributions}

All authors contributed to the development and writing of the manuscript and approved its final version.

\section{Funding}

None.

Availability of data and materials Not applicable.

Ethics approval and consent to participate Not applicable.

Consent for publication

Not applicable.

\section{Competing interests}

$\mathrm{KH}$ has received honoraria from AstraZeneca K.K. and Ono Pharmaceutical Co. Ltd.; lecture fees from AS ONE Corp., AstraZeneca K.K., Bristol-Myers Squibb Co. Ltd., Chugai Pharmaceutical Co. Ltd., MSD K.K., Ono Pharmaceutical Co. Ltd., and Pfizer Japan Inc.; and research funding from AstraZeneca K.K. HH has received honoraria from AstraZeneca K.K., Boehringer Ingelheim Japan Inc., Bristol-Myers Squibb Co. Ltd., Chugai Pharmaceutical Co. Ltd., Eli Lilly Japan K.K., Kyorin Pharmaceutical Co. Ltd., MSD K.K., Ono Pharmaceutical Co. Ltd., Pfizer Japan Inc., Shanghai Haihe Biopharm, and Taiho Pharmaceutical Co. Ltd. as well as research funding from AstraZeneca K.K., Boehringer Ingelheim Japan Inc., and Ono Pharmaceutical Co. Ltd. KN has received honoraria from Astellas Pharma Inc., AstraZeneca K.K., Bristol-Myers Squibb Co. Ltd., Chugai Pharmaceutical Co. Ltd., Clinical Trial Co. Ltd., Eli Lilly Japan K.K., MSD K.K., Nichi-Iko Pharmaceutical Co. Ltd., Nippon Boehringer Ingelheim Co. Ltd., Novartis Pharma K.K., Ono Pharmaceutical Co. Ltd., Pfizer Japan Inc., Reno Medical K.K., and Sym Bio Pharmaceuticals Ltd.; research funding from A2 Healthcare Corp., AbbVie Inc., Astellas Pharma Inc., Bristol-Myers Squibb Co. Ltd., Chugai Pharmaceutical Co. Ltd., Daiichi Sankyo Co. Ltd., Eisai Co. Ltd., Eli Lilly Japan K.K., EP-CRSU Co. Ltd., Gritstone Oncology Inc., ICON Japan K.K., inVentiv Health Japan, MSD K.K., Linical Co. Ltd., Nippon Boehringer Ingelheim Co. Ltd., Novartis Pharma K.K., Ono Pharmaceutical Co. Ltd., Parexel International Corp., Pfizer Japan Inc., Quintiles Inc., Taiho Pharmaceutical Co. 
Ltd., and Takeda Pharmaceutical Co. Ltd.; and consulting or advisory fees from Astellas Pharma Inc., Takeda Pharmaceutical Co. Ltd., and Ono Pharmaceutical Co. Ltd.

Received: 31 March 2020 Accepted: 1 April 2020

Published online: 21 April 2020

\section{References}

1. Ribas A, Wolchok JD. Cancer immunotherapy using checkpoint blockade. Science. 2018;359(6382):1350-5.

2. Postow MA, Sidlow R, Hellmann MD. Immune-related adverse events associated with immune checkpoint blockade. N Engl J Med. 2018;378(2): 158-68.

3. Das S, Johnson DB. Immune-related adverse events and anti-tumor efficacy of immune checkpoint inhibitors. J Immunother Cancer. 2019:7(1):306

4. Zhou X, Yao Z, Yang H, Liang N, Zhang X, Zhang F. Are immune-related adverse events associated with the efficacy of immune checkpoint inhibitors in patients with cancer? A systematic review and meta analysis. BMC Med. 2020. https://doi.org/10.1186/s12916-020-01549-2.

5. Stang A. Critical evaluation of the Newcastle-Ottawa scale for the assessment of the quality of nonrandomized studies in meta-analyses. Eur J Epidemiol. 2010;25(9):603-5.

6. Haratani K, Hayashi H, Chiba Y, Kudo K, Yonesaka K, Kato R, Kaneda H, Hasegawa Y, Tanaka K, Takeda M, et al. Association of immune-related adverse events with nivolumab efficacy in non-small-cell lung cancer. JAMA Oncol. 2018;4(3):374-8.

7. Naqash AR, Ricciuti B, Owen DH, Florou V, Toi Y, Cherry C, Hafiz M, De Giglio A, Muzaffar M, Patel SH, et al. Outcomes associated with immunerelated adverse events in metastatic non-small cell lung cancer treated with nivolumab: a pooled exploratory analysis from a global cohort. Cancer Immunol Immunother. 2020.

8. Tokunaga A, Sugiyama D, Maeda Y, Warner AB, Panageas KS, Ito S, Togashi Y, Sakai C, Wolchok JD, Nishikawa H. Selective inhibition of low-affinity memory CD8(+) T cells by corticosteroids. J Exp Med. 2019;216(12):2701-13.

9. Mustafa RA, Santesso N, Brozek J, Akl EA, Walter SD, Norman G, Kulasegaram M, Christensen R, Guyatt GH, Falck-Ytter Y, et al. The GRADE approach is reproducible in assessing the quality of evidence of quantitative evidence syntheses. J Clin Epidemiol. 2013;66(7):736-42 quiz 742 e731-735.

10. Schünemann $H$, Brożek J, Guyatt $G$, Oxman A, editors.: GRADE handbook for grading quality of evidence and strength of recommendations. Updated October 2013. The GRADE Working Group, 2013. Available from guidelinedevelopment.org/handbook.

\section{Publisher's Note}

Springer Nature remains neutral with regard to jurisdictional claims in published maps and institutional affiliations.

Ready to submit your research? Choose BMC and benefit from:

- fast, convenient online submission

- thorough peer review by experienced researchers in your field

- rapid publication on acceptance

- support for research data, including large and complex data types

- gold Open Access which fosters wider collaboration and increased citations

- maximum visibility for your research: over $100 \mathrm{M}$ website views per year

At BMC, research is always in progress.

Learn more biomedcentral.com/submissions 